
\title{
R Research S Surare \\ Diagnostic value of systematic ultrasonography for trisomy 18 syndrome in fetuses before 16 weeks gestation
}

Jie Zhou

West China Women \'s and Children \'s Hospital: Sichuan University West China Second University Hospital https://orcid.org/0000-0002-5104-0921

Xiaohui Dai

Hanmin Liu

Yiping Li

Ling Luo

Jiao Chen ( $\sim$ chenjiao@scu.edu.cn )

\section{Research Article}

Keywords: Early systematic ultrasonography, Structural malformation, Trisomy 18, Prenatal diagnosis

Posted Date: December 22nd, 2021

DOI: https://doi.org/10.21203/rs.3.rs-1139778/v1

License: (1) This work is licensed under a Creative Commons Attribution 4.0 International License.

Read Full License 


\section{Abstract \\ Purpose}

To explore the diagnostic value of systematic fetal ultrasonography for trisomy 18 (T18) syndrome before 16 weeks gestation.

\section{Methods}

A total of 12 fetuses with T18 were selected as research subjects and their nuchal translucency (NT) screening and fetal systematic ultrasonographic images acquired at 11-15 weeks were retrospectively analyzed.

\section{Results}

In the 12 fetuses' NT screening, ten fetuses showed NT thickening, one showed nuchal cystic hygroma, four showed reversed a-wave ductus venosus flow, and three showed omphalocele. The most common anomalies on the systematic ultrasonography before 16 weeks gestation were cardiac defects $(12 / 12$, $100 \%)$, omphalocele (4/12, 33.3\%), limb anomalies (5/12, 41.7\%), and facial anomalies $(3 / 12,25.0 \%)$. Seven of the 12 fetuses had multiple structural malformations: three had two structural malformations (25.0\%), three had three structural malformations (25.0\%), and one had four structural malformations $(8.3 \%)$.

\section{Conclusion}

Systematic fetal ultrasonography before 16 weeks gestation can detect most of the structural malformations of T18, effectively shortening the prenatal diagnosis time. It is therefore of great importance for reducing the birth rate of children with T18 and minimizing the physical and mental damage to mothers and their families.

\section{Introduction}

The early detection of abnormal fetal development on prenatal ultrasonography screening has become the consensus action of experts worldwide. With improvement in the resolution of ultrasonography instrumentation, more structures can be detected on early fetal ultrasound, and early ultrasonography has been widely introduced into antenatal care. This has resulted in more fetuses being identified with serious congenital abnormalities at an early stage. Trisomy 18 (T18) is the second most common trisomy after trisomy 21 syndrome, with an overall prevalence of 1/2500. It is often associated with multiple congenital anomalies and shows a preference for female fetuses. Abortion and pre-natal mortality account for up to $80 \%$ of cases, while approximately $90 \%$ of newborns do not survive beyond 1 year of age [1-3]. 
Ultrasonography is not only an important part of clinical management, but may also affect the choice of confirmatory prenatal diagnostic procedure $[3,4]$. It is thus of great significance in the early diagnosis of the disease.

The purpose of this study was to summarize the early sonographic manifestations of T18 in fetuses confirmed by chromosome examination, and to explore the value of systematic early fetal ultrasonography in the diagnosis of T18 syndrome.

\section{Methods}

\subsection{Research Subjects}

The study population consisted of 12 fetuses with T18 that showed abnormal results on systematic early ultrasonography. The pregnant women had a mean age of 26.6 (19-33) years and presented at our center between 2020 and 2021. T18 was confirmed by amniocentesis $(n=5)$ or genome-wide CNV analysis following induced labor $(n=7)$. Eight of the fetuses were diagnosed as $G 1 P 0$, three as G2P0, and one as G4P1A2L1. Three of the pregnant women had a history of adverse pregnancy. The mean gestational age was $14\left(12^{+2}-15^{+4}\right)$ weeks, six fetuses were male, and six were female.

\subsection{Instruments and Methods}

Sonographic examinations were performed using an Aplio 500 system (Canon Medical Systems Corporation, Tochigi, Japan) with transabdominal US (3-10 MHz). Transvaginal US (6-12 MHz) was used only if required to complete the scan. The scan protocol consisted of two parts, according to the published guidelines [5-8]. First, a nuchal translucency (NT) scan was performed, which included measurement of NT and evaluation of secondary markers for the presence of nasal bone, tricuspid regurgitation, and flow in the ductus venosus (DV). Second, systematic early fetal ultrasonography including an early simplified fetal echocardiographic examination was performed. This included a detailed screening for anatomical anomalies including continuity of the calvarium, division of cerebral hemispheres, presence of orbits, continuity of the secondary palate in the axial view, relationships of thoracic organs, the cardiac axis, four-chamber view, three-vessel and trachea view, position of the stomach in the abdominal cavity, abdominal wall continuity, presence and size of the urinary bladder, number of umbilical arteries, and upper and lower limbs. All materials and data were stored on the workstations.

\subsection{Statistical analysis}

Count data are expressed as percentage (\%). Measurement data with a normal distribution are expressed as mean, whereas data with a non-normal distribution are expressed as median with interquartile range.

\section{Results}


In the NT screenings, the crown-rump lengths of the 12 fetuses ranged from 4.74 to $7.79 \mathrm{~mm}$, and the gestational age ranged from $11^{+4}$ to $13^{+4}$ weeks. The mean NT was $4.6 \mathrm{~mm}(1.6-8.6 \mathrm{~mm})$, and the median fetal heart rate was 169 beats per minute (159-198bpm). Ten fetuses showed NT thickening, two showed nuchal cystic hygroma, five showed abnormal a-wave in ductus venosus flow, three showed omphalocele. (Table 1).

The mean gestational age according to systematic ultrasonography was $14^{+2}\left(12^{+2}-15^{+4}\right)$ weeks. All 12 fetuses showed varying degrees of cardiac malformations (100\%), including seven cases of ventricular septal defect (VSD, 58.3\%), two cases of double outlet right ventricle (DORV, 16.7\%), one case of truncus arteriosus (8.3\%), one case of isolated levocardia (8.3\%), three cases of coarctation of the aorta (COA) (figure $1 \mathrm{a}, \mathrm{b})(25.0 \%)$, one case of transposition of the great arteries (8.3\%), and one case of complete atrioventricular septal defect (8.3\%). Four fetuses showed abdominal anomalies of omphalocele (figure $2 a, b)(33.3 \%)$, one of them accompanied by umbilical cord cyst. Five fetuses showed limb anomalies $(41.7 \%)$, with one case of right club foot, one case of radial agenesis and ulnar and metacarpal hypoplasia with abnormal position, one case of bilateral absent radius and heteroplasia of both hands (figure $3 a, b)$, one case of abnormal position of both wrists, and one case of absence of right radius with bilateral club foot. Three fetuses showed facial anomalies (25.0\%), with two cases of micrognathia and one case of bilateral cleft lip and complete cleft palate (figure 4a, b), micrognathia, and unilateral accessory ear. Among the 12 fetuses, seven showed structural malformations other than cardiac malformations, including three fetuses with two structural malformations (25.0\%), three with three structural malformations (25.0\%), and one with four structural malformations (8.3\%; Table 1).

\section{Discussion}

T18 is the second most common aneuploidy after trisomy 21 syndrome (T21), epidemiological studies have shown that the disease has a high mortality, with abortion and prenatal mortality rates adding up to $80 \%$ [1], a live birth prevalence ranging from 1/10000 to 1/3000 [2], and a survival rate for 1 year less than $10 \%$ [3]. There is a predominance of affected females, with a male to female ratio of $1: 3[1,3]$, however, we report a 1:1 frequency of sexes, which may be a result of the small sample size.

It is generally believed that the embryological basis of T18 is the non-separation of chromosomes during egg meiosis, which is closely related to advanced-age pregnancy [9]. In our study, the maternal ages were all under 35 years, with the oldest being 33 years. Most of the women were in their first pregnancy, suggesting that antenatal screening in pregnant women under 35 years-of-age should not be ignored.

Current prenatal fetal chromosomal abnormality screening tests include Down's syndrome (DS), noninvasive prenatal testing (NIPT), and amniocentesis. Although DS screening and NIPT, which can predict chromosomal abnormalities, are more commonly performed because of their non-invasive nature, it is mandatory to confirm a positive DS screening or NIPT result with invasive procedures such as amniocentesis, which can provide diagnostic confirmation. Research has shown a detection rate for DS screening of $40 \%$ (2.00\% false positive rate), with the addition of a T18 algorithm increasing the detection 
rate of T18 with an increase in the false positive rate [10]. The diagnostic accuracy of a single NIPT for T18 is limited, and its price is relatively high. The positive predictive value of NIPT is lower for T18 than for T21 [11]. Therefore, a combination of screening tests is recommended to increase the detection rate of aneuploidy, with ultrasonography forming a very important part. Although ultrasonography cannot directly detect the karyotype, fetuses with abnormal chromosomes tend to show congenital structural anomalies that can be detected on ultrasonography $[12,13]$. Since the $1980 \mathrm{~s}$, many researchers have tried to summarize the performance and value of ultrasound in the diagnosis of T18 [14], but most research focused on the second or third trimester [15-17], with relatively few studies reporting on the detection of T18 before this stage of gestation. With improvement in the resolution of instruments and progress in ultrasonic technology, systematic fetal ultrasonography is gradually emerging, resulting in the detection of more fetal congenital anomalies before 16 weeks gestation $[1,13,18]$.

Research shows that $95 \%$ of fetuses with T18 have at least one abnormal prenatal ultrasound finding [16]. Lai et al. [19] stated that the detection rate of ultrasonography for T18 was $92.7 \%$ before 14 weeks and $100 \%$ at $18-21$ weeks. In our study, more than $90 \%$ of cases showed NT thickening at $11-13^{+6}$ weeks gestation, and fetal structural abnormalities were detected in $100 \%$ of cases at $14-15^{+6}$ weeks gestation. Only one case showed no abnormal sonographic finding before 14 weeks of gestation, but a cardiac malformation was detected at 15 weeks gestation. These results show that structural abnormalities in T18 fetuses can be detected by systematic ultrasonography at $11-15^{+6}$ weeks.

Fetal NT measurement is an important method for screening for T18 in early pregnancy, and many studies on large samples of the general population have shown that the mean NT thickness in euploid fetuses is $1.7-1.8 \mathrm{~mm}[4,13]$. T18 fetuses usually show increased NT thickening $[4,13]$, and in our study, 9/12 fetuses (75.0\%) showed NT thickening and two fetuses showed nuchal cystic hygroma. Tang et al. [20] stated that when abnormal NT and DV were observed concomitantly, the probability of any trisomy was higher than that with NT alone. In our study, five fetuses showed NT thickening and abnormal DV, and abnormal DV might be associated with serious cardiac malformations [21].

Cardiac malformations are the most common structural abnormalities in T18 fetuses [22, 23], at 11-13 weeks gestation, extracardiac and cardiac malformations were identified in $35.1 \%$ and $70.3 \%$ of T18 fetuses, respectively [13]. In our study, cardiac malformations were detected in all fetuses (100\%), with these being in combination with extracardiac malformations in 7 of the 12 cases (58.3\%). Yang et al. [24] stated that the most sensitive ultrasonographic finding for T18 was increased NT under 16 weeks of gestation, but cardiac defect after 16 weeks gestation. The NT results in our study are consistent with previous reports, although cardiac malformations were detected in all 12 fetuses before 16 weeks gestation, indicating that an earlier detection time for fetal cardiac malformations may help to shorten the detection time window for T18 fetuses. Many studies have found that chromosome abnormalities are highly associated with congenital cardiac malformations [13, 22-24], with the most common malformation being VSD $[13,23]$. However, although VSD is the most frequent cardiac malformation observed in T18 fetuses, there is a high risk of a false-positive result if it is used to predict T18 [13, 22]. Wiechec et al. [13] found that a right dominant heart (RDH) including DORV, COA, mitral atresia, and 
hypoplastic left heart syndrome (HLHS) was more predictive of T18 than VSD. In our subjects, five fetuses showed RDH, including two cases of DORV and three cases of COA, and we suggest taking particular care to rule out T18 when fetal RDH is detected.

Sepulveda et al. [18] found that omphalocele was the most common detected abnormality before 14 weeks of gestation. The incidence of omphalocele on the first trimester was only second to that of cardiac malformation in our study, with three cases $(3 / 12,25.0 \%)$ detected before 14 weeks gestation and four cases $(4 / 12,33.3 \%)$ detected before 16 weeks gestation. This shows that omphalocele is an important diagnostic clue, especially in combination with cardiac abnormalities, where the risk of T18 is increased.

While overlapping fingers and clenched or closed hands are characteristic manifestations of skeletal dysplasia in T18, other deformities such as abnormal foot posture, short limb deformity, and absence or dysplasia of the radius can also be detected in fetuses with T18 [25]. In our study, five cases (5/12, $41.7 \%$ ) of limb abnormalities were detected, with the incidence of limb abnormalities being only second to that of cardiac malformation, and the incidence being higher than that reported by Wiechec et al. [13]. This higher incidence might be related to the gestational age of our subjects, which was a little later than the first trimester, and which is more conducive to the detection of fetal limb abnormalities. In our study, the limb abnormalities of T18 fetuses were mainly upper limb skeletal abnormalities, similar to those reported by Rice et al. [26].

Facial anomalies are also common noticeable ultrasound markers for T18 fetuses, with an incidence of $14.3-41.5 \%[23,27]$. The most common facial deformities are micrognathia, followed by cleft lip and palate, and ear abnormalities, with the incidence of eye and nose deformities being low [27]. In our study, the main facial deformities were micrognathia, including one case accompanied by bilateral cleft lip, complete cleft palate, and unilateral accessory ear, indicating that relevant chromosome examinations should be carried out when such facial deformities are detected.

Nervous system anomalies are common ultrasonography-detectable malformations of T18 in the second and third trimester ${ }^{23}$, with an incidence of $12.9-21.6 \%[23,27]$ for abnormal cranial bone configuration (including brachycephaly, strawberry-shaped skull, and microcephaly). However, no nervous system abnormalities were found in our study, which might be a result of the young observational gestational age, at which nervous system development was incomplete, with some abnormalities not being obvious at this early stage.

Soft markers are fetal prenatal sonographic findings of microstructural abnormalities that are nonspecific, often transient, and differ from fetal malformations. In themselves they do not affect fetal development, although they may be detected in normal fetuses, but they may also be associated with fetal chromosomal abnormalities and are considered as risk factors for underlying fetal aneuploidy [28]. The most widely examined soft markers are thickened nuchal fold, mild ventriculomegaly, choroid plexus cysts, enlarged cisterna magna, absent or hypoplastic nasal bone, echogenic intracardiac focus, aberrant 
right subclavian artery, echogenic bowel, mild hydronephrosis, single umbilical artery, and short femur length [29]. Some of these findings can be used as additional ultrasonic indicators to evaluate the possibility of T18 [28]. Soft markers were identified in three $(3 / 12,25.0 \%)$ fetuses in our study, including one case of choroid plexus cysts and two cases of single umbilical artery. These results suggest that soft makers should not be ignored, that they have a certain value for screening for $\mathrm{T} 18$, and that pregnant women should be advised to have prenatal eugenic counseling when a fetal soft marker is identified.

Previous studies found a variety of structural abnormalities in T18 fetuses $[13,18,23,27]$, the nondisjunction of the chromosomes in the process of cell division in T18 fetuses may result in impacts on multiple systems [9]. Among the 12 fetuses in our study, seven $(7 / 12,58.3 \%)$ showed multiple structural malformations, including three cases of two and three structural malformations, respectively, and one case of four structural malformations. Thus, when one structural malformation is detected in a fetus, attention should be paid to the exclusion of combined malformations. When multiple structural malformations are identified, the possibility of T18 should be carefully considered. In this study, the majority of multisite structural malformations were heart abnormalities combined with omphalocele, or heart abnormalities combined with limb abnormalities. We suggest that when these two types of combined malformations are found, the possibility of T18 should be carefully considered.

\section{Conclusions}

With improvement in the resolution of instruments and progress in ultrasonic technology, structural malformations of T18 fetuses that used to be found on second and third trimester ultrasonography can now be detected on early systematic ultrasonography before 16 weeks gestation, improving the detection of chromosome abnormalities. Ultrasonography is of great value for guiding the clinical selection of prenatal diagnostic procedures, reducing the birth rate of children with T18 syndrome, and minimizing the physical and mental damage to women (and their families) carrying such fetuses.

\section{Declarations}

Authors' contributions Zhou and Xiaohui Dai: manuscript draft, data analysis and interpretation. Hanmin Liu: project development. Yiping Li and Ling Luo: data collection. Jiao Chen: project design, picture processing, text revision.

Funding This work was sponsored by the National Key R\&D Program of China (2017YFC0211705, 2017YFC0113905), the Key R\&D Program of Science and Technology Department of Sichuan Province (2019YFS0403, 2019YFS0037), and the Popularization and Application Project of the Sichuan Health and Family Planning Commission (17PJ41)

\section{Declarations}

Conflict of interest The authors declare that they have no competing interests. 
Ethical approval Ethical approval was obtained from the Institutional Ethics Committee of West China Second University Hospital of Sichuan University. genome-wide CNV analysis following induced labor was performed after obtaining the parents' informed consent.

Informed consent Informed consent was obtained from all individual participants included in the study.

\section{References}

1. Triunfo, S., Bonollo, M., Gaffuri, P., Viviano, M., Satta, D., \& Bergmann, M. (2021). A visual tool inclusive of fetal ultrasound and autopsy findings to reach a balanced approach to counseling on trisomy 18 in early second trimester. Archives of gynecology and obstetrics, 304(5), 1115-1125.

https://doi.org/10.1007/s00404-021-06130-7. 2. Imataka, G., Suzumura, H., \& Arisaka, O. (2016). Clinical features and survival in individuals with trisomy 18: A retrospective one-center study of 44 patients who received intensive care treatments. Molecular medicine reports, 13(3), 2457-2466.

https://doi.org/10.3892/mmr.2016.4806 3. Nagase, H., Ishikawa, H., Toyoshima, K., Itani, Y., Furuya, N., Kurosawa, K., Hirahara, F., \& Yamanaka, M. (2016). Fetal outcome of trisomy 18 diagnosed after 22 weeks of gestation: Experience of 123 cases at a single perinatal center. Congenital anomalies, 56(1), 35-40. https://doi.org/10.1111/cga.12118 4. Wagner, P., Sonek, J., Hoopmann, M., Abele, H., \& Kagan, K. O. (2016). First-trimester screening for trisomies 18 and 13, triploidy and Turner syndrome by detailed early anomaly scan. Ultrasound in obstetrics \& gynecology: the official journal of the International Society of Ultrasound in Obstetrics and Gynecology, 48(4), 446-451. https://doi.org/10.1002/uog.15829 5. Von Kaisenberg, C., Chaoui, R., Häusler, M., Kagan, K. O., Kozlowski, P., Merz, E., Rempen, A., Steiner, H., Tercanli, S., Wisser, J., \& Heling, K. S. (2016). Quality Requirements for the early Fetal Ultrasound Assessment at 11-13+6 Weeks of Gestation (DEGUM Levels II and III). Qualitätsanforderungen an die weiterführende differenzierte Ultraschalluntersuchung in der pränatalen Diagnostik (DEGUM-Stufen II und III) im Zeitraum 11-13+6 Schwangerschaftswochen. Ultraschall in der Medizin (Stuttgart, Germany: 1980), 37(3), 297-302. https://doi.org/10.1055/s-0042-105514 6. Kagan, K. O., Cicero, S., Staboulidou, I., Wright, D., \& Nicolaides, K. H. (2009). Fetal nasal bone in screening for trisomies 21, 18 and 13 and Turner syndrome at 11-13 weeks of gestation. Ultrasound in obstetrics \& gynecology: the official journal of the International Society of Ultrasound in Obstetrics and Gynecology, 33(3), 259-264. https://doi.org/10.1002/uog.6318 7. Kagan, K. O., Valencia, C., Livanos, P., Wright, D., \& Nicolaides, K. H. (2009). Tricuspid regurgitation in screening for trisomies 21,18 and 13 and Turner syndrome at $11+0$ to $13+6$ weeks of gestation. Ultrasound in obstetrics \& gynecology: the official journal of the International Society of Ultrasound in Obstetrics and Gynecology, 33(1), 18-22. https://doi.org/10.1002/uog.6264 8. Maiz, N., Valencia, C., Kagan, K. O., Wright, D., \& Nicolaides, K. H. (2009). Ductus venosus Doppler in screening for trisomies 21,18 and 13 and Turner syndrome at 11-13 weeks of gestation. Ultrasound in obstetrics \& gynecology: the official journal of the International Society of Ultrasound in Obstetrics and Gynecology, 33(5), 512-517. https://doi.org/10.1002/uog.6330 9. Rosa, R. F., Rosa, R. C., Zen, P. R., Graziadio, C., \& Paskulin, G. A. (2013). Trisomy 18: review of the clinical, etiologic, prognostic, and ethical aspects. Revista paulista de pediatria : orgao oficial da Sociedade de Pediatria de Sao Paulo, 31(1), 111120. https://doi.org/10.1590/s0103-05822013000100018 10. Bestwick, J. P., Huttly, W. J., \& Wald, N. J. 
(2013). Detection of trisomy 18 and trisomy 13 using first and second trimester Down's syndrome screening markers. Journal of medical screening, 20(2), 57-65.

https://doi.org/10.1177/0969141313484904 11. Zhen, L., Yang, Y. D., Li, Y. J., Xu, L. L., \& Li, D. Z. (2019). The role of ultrasound in the choice between chorionic villus sampling and amniocentesis for patients with a positive NIPT result for trisomy 18/13. Prenatal diagnosis, 39(12), 1155-1158.

https://doi.org/10.1002/pd.5524 12. Jelin, A., Sagaser, K., Lawson, C., Forster, K. R., Leppert, K., Hertenstein, C., Batista, D., \& Blakemore, K. (2019). Chromosomal Microarray Analysis Results From Pregnancies With Various Ultrasonographic Anomalies. Obstetrics and gynecology, 133(4), 827-828. https://doi.org/10.1097/AOG.0000000000003207 13. Wiechec, M., Knafel, A., Nocun, A., Matyszkiewicz, A., Wiercinska, E., \& Latała, E. (2016). How effective is ultrasound-based screening for trisomy 18 without the addition of biochemistry at the time of late first trimester? Journal of perinatal medicine, 44(2), 149159. https://doi.org/10.1515/jpm-2014-0384 14. Kurjak, A., \& Kirkinen, P. (1982). Ultrasonic growth pattern of fetuses with chromosomal aberrations. Acta obstetricia et gynecologica Scandinavica, 61(3), 223-225. https://doi.org/10.3109/00016348209156561 15. Vos, F. I., de Jong-Pleij, E. A., Bakker, M., Tromp, E., Manten, G. T., \& Bilardo, C. M. (2015). Facial profile markers in second- and third-trimester fetuses with trisomy 18. Ultrasound in obstetrics \& gynecology: the official journal of the International Society of Ultrasound in Obstetrics and Gynecology, 46(1), 66-72. https://doi.org/10.1002/uog.14662 16. Becker, D. A., Tang, Y., Jacobs, A. P., Biggio, J. R., Edwards, R. K., \& Subramaniam, A. (2019). Sensitivity of prenatal ultrasound for detection of trisomy 18 . The journal of maternal-fetal \& neonatal medicine: the official journal of the European Association of Perinatal Medicine, the Federation of Asia and Oceania Perinatal Societies, the International Society of Perinatal Obstetricians, 32(22), 3716-3722. https://doi.org/10.1080/14767058.2018.1471460 17. Nagase, H., Ishikawa, H., Toyoshima, K., Itani, Y., Furuya, N., Kurosawa, K., Hirahara, F., \& Yamanaka, M. (2016). Fetal outcome of trisomy 18 diagnosed after 22 weeks of gestation: Experience of 123 cases at a single perinatal center. Congenital anomalies, 56(1), 35-40. https://doi.org/10.1111/cga.12118 18. Sepulveda, W., Wong, A. E., \& Dezerega, V. (2010). First-trimester sonographic findings in trisomy 18: a review of 53 cases. Prenatal diagnosis, 30(3), 256259. https://doi.org/10.1002/pd.2462 19. Lai, S., Lau, W. L., Leung, W. C., Lai, F. K., \& Chin, R. (2010). Is ultrasound alone enough for prenatal screening of trisomy 18 ? A single centre experience in 69 cases over 10 years. Prenatal diagnosis, 30(11), 1094-1099. https://doi.org/10.1002/pd.2623 20. Tang, Y., Luo, H., Mu, D., Yang, T., Zhu, Q., Yang, F., \& Liu, G. (2019). Early diagnosis of trisomy 21, trisomy 18 and trisomy 13 using nuchal translucency thickness and ductus venosus blood flow waveform in West China. Molecular medicine reports, 19(2), 1349-1355. https://doi.org/10.3892/mmr.2018.9756 21. Wagner, P., Eberle, K., Sonek, J., Berg, C., Gembruch, U., Hoopmann, M., Prodan, N., \& Kagan, K. O. (2019). Firsttrimester ductus venosus velocity ratio as a marker of major cardiac defects. Ultrasound in obstetrics \& gynecology: the official journal of the International Society of Ultrasound in Obstetrics and Gynecology, 53(5), 663-668. https://doi.org/10.1002/uog.20099 22. Lin, A. E., Santoro, S., High, F. A., Goldenberg, P., \& Gutmark-Little, I. (2020). Congenital heart defects associated with aneuploidy syndromes: New insights into familiar associations. American journal of medical genetics. Part C, Seminars in medical genetics, 184(1), 53-63. https://doi.org/10.1002/ajmg.c.31760 23. Papp, C., Ban, Z., Szigeti, Z., Csaba, A., Beke, A., \& Papp, Z. (2007). Role of second trimester sonography in detecting trisomy 18: a review of 70 cases. 
Journal of clinical ultrasound: JCU, 35(2), 68-72. https://doi.org/10.1002/jcu.20290 24. Yang, J. H., Chung, J. H., Shin, J. S., Choi, J. S., Ryu, H. M., \& Kim, M. Y. (2005). Prenatal diagnosis of trisomy 18: report of 30 cases. Prenatal diagnosis, 25(2), 119-122. https://doi.org/10.1002/pd.1073 25. Viora, E., Zamboni, C., Mortara, G., Stillavato, S., Bastonero, S., Errante, G., Sciarrone, A., \& Campogrande, M. (2007). Trisomy 18: Fetal ultrasound findings at different gestational ages. American journal of medical genetics. Part A, 143A(6), 553-557. https://doi.org/10.1002/ajmg.a.31615 26. Rice, K. J., Ballas, J., Lai, E., Hartney, C., Jones, M. C., \& Pretorius, D. H. (2011). Diagnosis of fetal limb abnormalities before 15 weeks: cause for concern. Journal of ultrasound in medicine: official journal of the American Institute of Ultrasound in Medicine, 30(7), 1009-1019. https://doi.org/10.7863/jum.2011.30.7.1009 27. Ettema, A. M., Wenghoefer, M., Hansmann, M., Carels, C. E., Borstlap, W. A., \& Bergé, S. J. (2010). Prenatal diagnosis of craniomaxillofacial malformations: a characterization of phenotypes in trisomies 13, 18, and 21 by ultrasound and pathology. The Cleft palate-craniofacial journal: official publication of the American Cleft Palate-Craniofacial Association, 47(2), 189-196. https://doi.org/10.1597/08-285_1 28. Czuba, B., Maczka, M., Cnota, W., Wloch, A., Jagielska, A., Niesluchowska-Hoxha, A., \& Borowski, D. (2020). Nasal bone in screening for Trisomy 18 and 13 at 11-13 6 weeks of gestation - own experiences. Ginekologia polska, 91(5), 256-261. https://doi.org/10.5603/GP.2020.0047 29. Hu, T., Tian, T., Zhang, Z., Wang, J., Hu, R., Xiao, L., Zhu, H., Lai, Y., Wang, H., \& Liu, S. (2021). Prenatal chromosomal microarray analysis in 2466 fetuses with ultrasonographic soft markers: a prospective cohort study. American journal of obstetrics and gynecology, 224(5), 516.e1-516.e16. https://doi.org/10.1016/j.ajog.2020.10.039

\section{Figures}

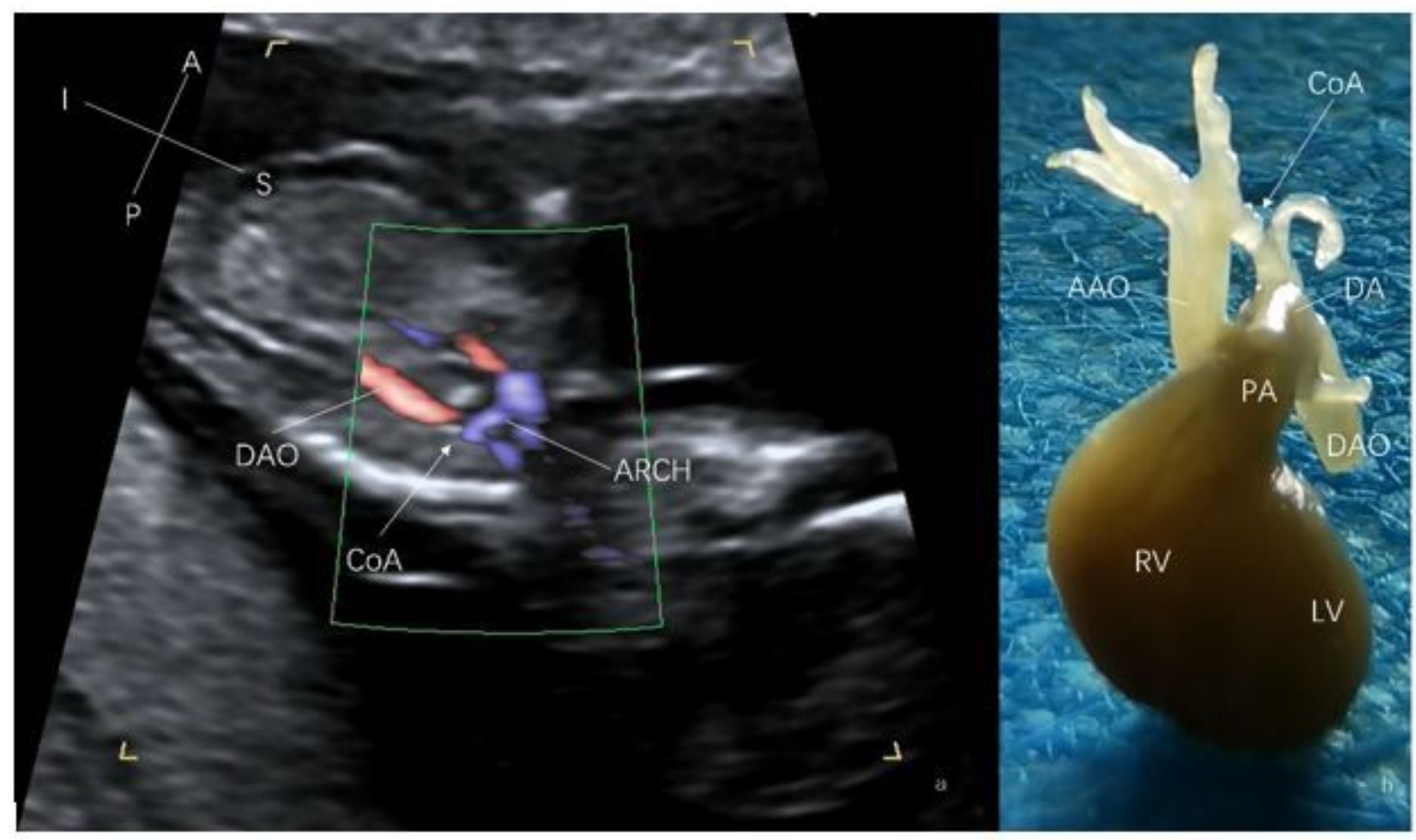

Figure 1 
Fetal coarctation of aorta. a. Color Doppler flow imaging of prenatal fetal ultrasonography showing significant narrowing of the descending aortic tract on the long axis section of the aortic arch (arrow). b. Gross specimen of the induced fetal heart showing coarctation of aorta (arrow).

$\mathrm{ARCH}$, aortic arch; CoA, coarctation of aorta; DAO, descending aorta; LV, left ventricle; RV, right ventricle; $P A$, pulmonary artery; $A A O$, ascending aorta; $D A$, ductus arteriasus; $A$, anterior; $P$, posterior; $R$, right; $L$, left; $\mathrm{S}$, superior; I, inferior.

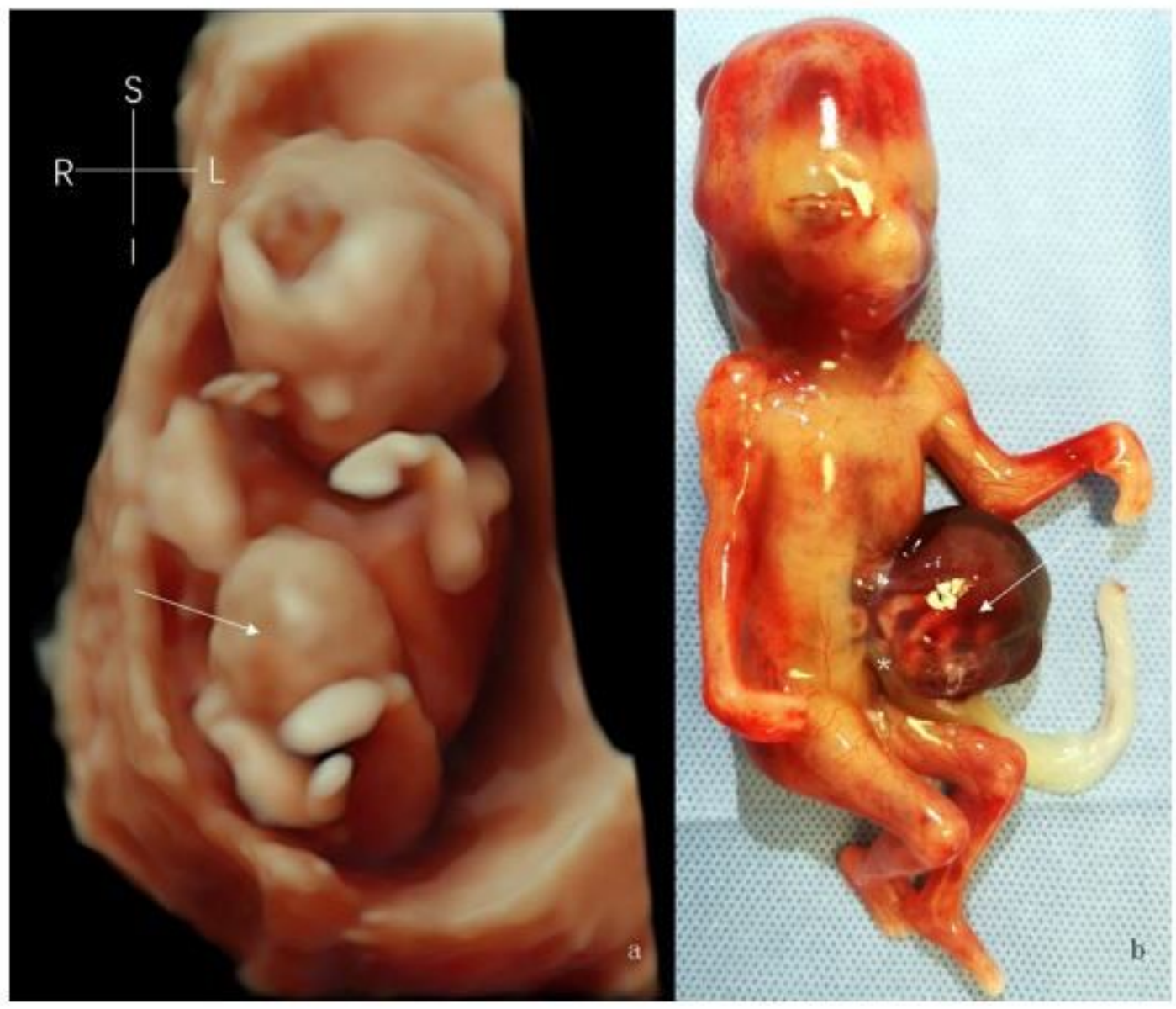

\section{Figure 2}

Fetal omphalocele. a. Three-dimensional surface imaging model of prenatal fetal ultrasonography showing fetal abdominal mass (arrow). b. Gross specimen of the induced fetus showing omphalocele (arrow) covered by a capsule with umbilical cord $\left(^{*}\right)$ attached to it.

$R$, right; L, left; S, superior; I, inferior. 


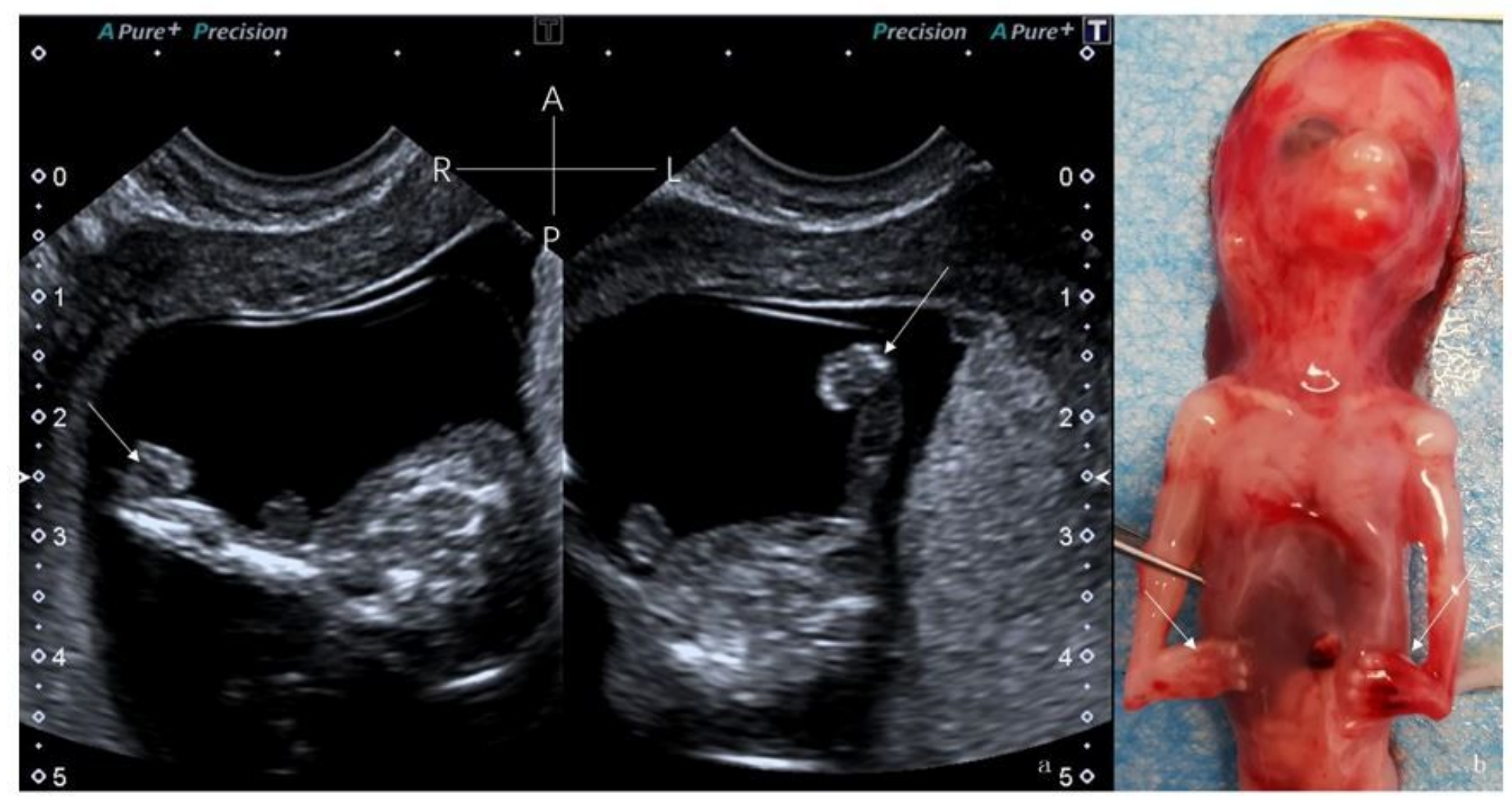

\section{Figure 3}

Fetal absent radius and short ulna. a. Two-dimensional ultrasound of prenatal fetal ultrasonography showing absent radius adjacent to a shortened ulna. The hand (arrow) is rotated inward to lie adjacent to the shaft of the ulna. The humerus is normal. b. Gross specimen of the induced fetus showing the forearms on both sides of the upper limbs are short, the wrist is curved at an acute Angle to the radial side with the thumb absent (arrow).

$A$, anterior; $P$, posterior; $R$, right; $L$, left 


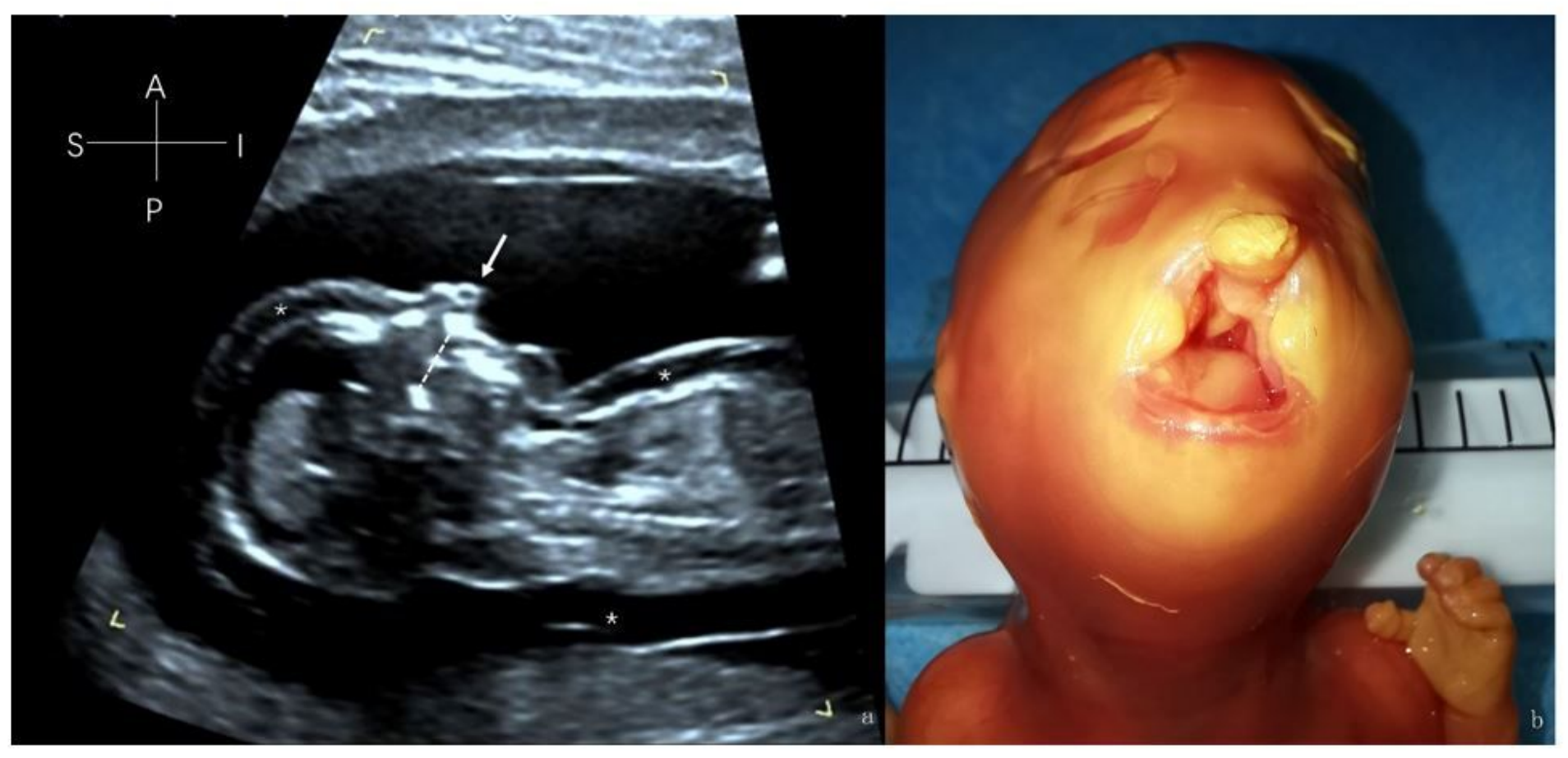

\section{Figure 4}

Fetal bilateral cleft lip with complete cleft palate. a. Two-dimensional ultrasound of prenatal fetal ultrasonography showing fetal maxillary projection (arrow), interrupted palatal continuity (dotted line), and skin edema $\left(^{\star}\right)$. b. Gross specimen of the induced fetus showing bilateral cleft lip and complete cleft palate.

R, right; L, left; S, superior; I, inferior. 